\title{
Iris Matching by Local Extremum Points of Multiscale Taylor Expansion
}

\author{
Algirdas Bastys, Justas Kranauskas, and Rokas Masiulis \\ Department of Computer Science II, \\ Faculty of Mathematics and Informatics, \\ Vilnius University, Lithuania
}

\begin{abstract}
Random distribution of features in iris image texture allows to perform iris-based personal authentication with high confidence. We propose to use the most significant local extremum points of the first two Taylor expansion coefficients as descriptors of the iris texture. A measure of similarity that is robust to moderate inaccuracies in iris segmentation is presented for the proposed features. We provide experimental results of verification quality for four commonly used iris data-sets. Strong and weak aspects of the proposed approach are also discussed.
\end{abstract}

\section{Introducation}

Person identification based on iris recognition gives one of the most reliable results. Iris texture features provides a unique high dimensional information that explains why iris recognition based verification has the lowest false acceptance rate among all types of biometric verification systems [1, [2]. A transparent and simple distribution of calculated distances between iris pairs allows to make a fine prediction of verification quality for a chosen distance threshold [3]. Large-scale application of iris recognition for border-crossing control showed up robustness of the technology even in case of more than half billion different iris images spanning about 150 nationalities [4. Appearance of new technologies that enable to capture iris images even at distance of 20 meters significantly reduced requirements for subject cooperation with the automated identification system [5].

In this paper, we focus on iris texture description and comparison, thus iris segmentation algorithm is left aside. However, our implementation of fully automatic segmentation algorithm, which approximates iris inner and outer boundaries by Fourier series and unwraps iris texture into polar like representation (similar to Daugman's rubber sheet method), was used in all presented experiments. Iris local features as local extrema of multiscale Taylor expansion are presented in section 2 Only approximations of Taylor coefficients associated with the first and second order derivatives averaged on different scales are used. The most significant local extrema of the expansions shows where the most significant iris texture variations are. In section 3, a metric for comparison of feature sets formed from the local extrema is presented. Quality of the proposed iris recognition algorithm is analyzed in section 4 which is followed by a discussion and conclusion sections.

M. Tistarelli and M.S. Nixon (Eds.): ICB 2009, LNCS 5558, pp. 1070-1079, 2009.

(C) Springer-Verlag Berlin Heidelberg 2009 


\section{Local Extrema of Multiscale Taylor Expansion}

We developed our scheme of iris representation by local features considering the following constraints: number of local features should be easily controlled and their attributes should be as compact and simple as possible. The main goal of our representation is to control memory consumption and iris matching speed, thus leading to the fast and robust iris-based verification and identification.

\subsection{Local Descriptors}

Let's consider one dimensional iris signal having a fixed pseudopolar radius $r=$ const as an analytic one-dimensional signal $u=u(x)$, where $x$ is pseudopolar angle. It is well known that any signal can be expressed in classic Taylor series expansion centered on any fixed $x=x_{i}$. The zero coefficient of the expansion equals to $u\left(x_{i}\right)$ and is strongly influenced by iris lighting conditions thus can not be a robust iris descriptor. The second two coefficients equal to $\frac{u^{\prime}\left(x_{i}\right)}{1 !}$ and $\frac{u^{\prime \prime}\left(x_{i}\right)}{2 !}$. The first derivative $u^{\prime}(x)$ estimates transition rate of the signal around point $x$, i.e. the big positive value of $u^{\prime}(x)$ means a fast increasing transition of the signal around point $x$ and a big negative $u^{\prime}(x)$ means a fast decay around the same point. The sign of the second derivative $u^{\prime \prime}(x)$ provides information about the type of concavity of graph of the transition around $x$, i.e. if $u^{\prime \prime}(x)>0$ then graph of transition of the signal is concave upward and $u^{\prime \prime}(x)<0$ gives that graph of the signal that is concave downward around point $x$. If transition of $x$ values corresponds to transition of $u=u(x)$ from left to right through a dark or bright blob one can expect the following transitions of the signs:

\begin{tabular}{|c|c|c|}
\hline Blob & Derivative & Transition of signs \\
\hline \multirow{2}{*}{ Dark } & $u^{\prime}(x)$ & -+ \\
& $u^{\prime \prime}(x)$ & -+- \\
\hline \multirow{2}{*}{ Bright } & $u^{\prime}(x)$ & +- \\
& $u^{\prime \prime}(x)$ & +-+ \\
\hline
\end{tabular}

Left and right boundary of a dark blob can be marked by local negative minimum and positive maximum of the first derivative. The second derivative will have zero crossings around these local extrema. If we expand such features to two-dimensional case, only a fraction of $u_{x x}(x, y)$ zero crossings will correspond to $u_{x}(x, y)$ local extrema. Therefore local extrema of both two-dimensional functions $u_{x}(x, y)$ and $u_{x x}(x, y)$ were used as primary source for derivation of iris local features. Notice that $u^{\prime}(x)$ and $u^{\prime \prime}(x)$ derivatives can be estimated only numerically in the context of digital iris data and the result of approximations significantly depends on scale at which increments of the signal are estimated. $u^{\prime \prime}(x)$ approximation will have a positive maximum close to a dark blob center and a negative minimum close to a bright blob center if the chosen scale resonates with blob size. 
The well known Taylor series expansion

$$
u(x)=\sum_{n=0}^{\infty} \frac{u^{(n)}(a)}{n !}(x-a)^{n}
$$

tells us that sufficiently regular function can be reconstructed from its Taylor coefficients $\frac{u^{(n)}(a)}{n !}$. In theory Taylor coefficients can be derived from $u(x)$ values known at any narrow surrounding of the center point $x=a$. Let us consider some details of estimation of local extrema of the first two derivatives of iris texture and conditions under which a particular extremum point is included in the set of iris local features. Estimation technique of derivatives at different scales is similar to the wavelet multiresolution analysis. Let us fix any symmetric (even) smooth filter $p=p(x) \geq 0$ which defines rule of derivatives averaging. Let us fix some scale $\sigma>0$ and consider convolutions

$$
\left(u^{\prime} * D_{\sigma} p\right)(x)=1 / \sigma \int_{-\infty}^{\infty} p(\xi / \sigma) u^{\prime}(\xi-x) d \xi
$$

and

$$
\left(u^{\prime \prime} * D_{\sigma} p\right)(x)=1 / \sigma \int_{-\infty}^{\infty} p(\xi / \sigma) u^{\prime \prime}(\xi-x) d \xi .
$$

In the bigger scale $\sigma$ the more blurred version of $u^{\prime}(x)$ and $u^{\prime \prime}(x)$ represents $\left(u^{\prime} * D_{\sigma} p\right)(x)$ and $\left(u^{\prime \prime} * D_{\sigma} p\right)(x)$. One the other hand, if $\sigma$ approaches zero then $\left(u^{\prime} * D_{\sigma} p\right)(x)$ and $\left(u^{\prime \prime} * D_{\sigma} p\right)(x)$ approaches to $u^{\prime}(x)$ and $u^{\prime \prime}(x)$ respectively.

We have discrete periodic sequence $u_{i}=u(i)$ of length $N X$ in case of iris texture. Defining $u(x)$ by linear interpolation $u(x)=u_{i}+(x-i)\left(u_{i+1}-u_{i}\right)$, $x \in[i, i+1)$, we will have

$$
u^{\prime \prime}(x)=\sum_{i=-\infty}^{\infty}\left(u_{i+1}-2 u_{i}+u_{i-1}\right) \delta(x-i)
$$

and

$$
\begin{aligned}
\left(u^{\prime \prime} * D_{\sigma} p\right)(x) & =\sum_{i=-\infty}^{\infty}\left(u_{i+1}-2 u_{i}+u_{i-1}\right) p\left(\frac{x+i}{\sigma}\right) / \sigma \\
& =\sum_{i=0}^{N X-1} \Delta u_{i} p_{\sigma}^{N X}(x+i) \\
& =\sum_{i=0}^{N X-1} u_{i} \Delta p_{\sigma}^{N X}(x+i) .
\end{aligned}
$$

Here $\delta(x)$ is Dirac's delta function,

$$
\Delta u_{i}=u(i+1)-2 u(i)+u(i-1),
$$

and

$$
p_{\sigma}^{N X}(x)=\frac{\sum_{i=-\infty}^{\infty} p\left(\frac{x+i * N X}{\sigma}\right)}{\sigma}
$$


Similarly, for the first derivative we have

$$
\begin{aligned}
\left(u^{\prime} * D_{\sigma} p\right)(j) & =-\sum_{i=0}^{N X-1} \Delta u_{i} q^{N X} \sigma(j+i), \\
q_{\sigma}^{N X}(x) & =\sum_{i=-\infty}^{\infty} 2 \operatorname{arctg}\left(\frac{x+i * N X}{\sigma}\right) .
\end{aligned}
$$

From symmetry of the filter function follows that (5) defines a filtration of 1D data $\left\{u_{i}\right\}$ by an even-symmetric filter and (10) defines an odd-symmetric filter. In wavelet terminology the even and odd filters has two and one vanishing moments respectively. The proposed filters has more freedom in choosing basic function $p^{\sigma}=p^{\sigma}(x)$ in comparison with dyadic wavelet filtration and do not have dyadic restrictions for the scale $\sigma$. To regularize the filtration results that at different scales $\sigma$ approximate second (even filters) or first (odd filters) order derivatives we used some smoothing in orthogonal direction by applying the same filter $p^{\sigma}=p^{\sigma}(y)$ with smaller scale value $\sigma$.

Table 1 presents scales, type of symmetry and direction which were used to calculate differences. The differences calculated along $x$ and $y$ directions correspond respectively to horizontal and vertical type filters. Derivatives along $x$ direction were estimated at 3 different scales while derivatives along $y$ direction were estimated only at 1 scale.

Table 1. Derivatives and averaging parameters used in derivation of multiscale Taylor expansion and local features

\begin{tabular}{|c|c|c|c|}
\hline $\begin{array}{c}\text { Scale and } \\
\text { filter type } \\
\text { index } s\end{array}$ & $\begin{array}{c}\text { Blurred } \\
\text { derivative }\end{array}$ & $\begin{array}{c}\sigma_{x}=\sigma \text { of } \\
\text { horizontal } \\
\text { direction }\end{array}$ & $\begin{array}{c}\sigma_{y}=\sigma \text { of } \\
\text { vertical } \\
\text { direction }\end{array}$ \\
\hline 0 & $u_{x}$ & $\frac{6}{8}$ & $\frac{1}{2}$ \\
1 & $u_{x}$ & $\frac{9}{8}$ & $\frac{1}{2}$ \\
2 & $u_{x}$ & $\frac{15}{8}$ & $\frac{1}{2}$ \\
3 & $u_{x x}$ & $\frac{9}{8}$ & $\frac{1}{2}$ \\
4 & $u_{x x}$ & $\frac{15}{8}$ & $\frac{1}{2}$ \\
5 & $u_{x x}$ & $\frac{24}{8}$ & $\frac{1}{2}$ \\
6 & $u_{y}$ & $\frac{1}{2}$ & $\frac{9}{8}$ \\
7 & $u_{y y}$ & $\frac{1}{2}$ & $\frac{15}{8}$ \\
\hline
\end{tabular}

\subsection{Significant Local Descriptors}

Blurred derivative may be considered as the coefficients of Taylor series expansion beside $(x-a) / 1$ ! and $(x-a)^{2} / 2$ ! terms estimated at different scales or coefficients of wavelet expansion calculated at different scales with asymmetric or symmetric mother wavelets that have one or two vanishing moments. It is well known that coefficients with highest squared values give the main contribution in approximation of original signal by the expansions. Thus, extrema or 
more exactly, positive local maximums and negative local minimums of blurred derivatives, are good candidates for local descriptors of iris texture. Additionally, we required that the local extremum at particular scale $\sigma_{0}$ exceeded the same derivative at the same position but with rougher scales $\sigma>\sigma_{0}$ (that conditioned more stable extrema). And the very last condition for including the extremum point to the set of local iris features was its magnitude. However, we decided to avoid thresholds in this place and used the following procedure for choosing extrema points (see fig. 1):

1. Divide iris texture annual along polar angle direction into 16 equal sectors.

2. Enumerate sectors clockwise starting at " 0 " hour.

3. Eliminate local extrema which are found in upper or lower lids. Two different approaches are used:

(a) Find a convex domain that includes only iris with pupil and excludes lids.

(b) Ignore sectors $0,1,2,13,14$, and 15 because iris texture in these sectors will likely be hidden by upper lid. Ignore sectors 7 and 8 because iris texture in these sectors will likely be hidden by lower lid.

4. Choose no more than $K$ biggest local extrema from each scale and each active sector.

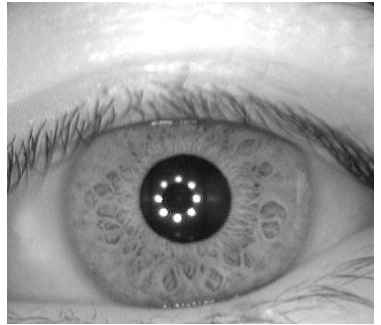

(a) Original image.

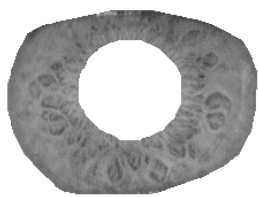

(b) A convex domain that includes only iris.

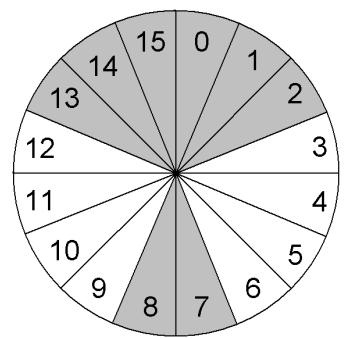

(c) Iris division into sectors.

Fig. 1. Elimination of local extrema which are found in upper or lower lids. In (c), active sectors are marked in white and inactive sectors are marked in light gray.

Figure 2 illustrates local extrema points that were found in active sectors for 242116.tiff and 241643.tiff iris images of NIST ICE2005 iris data-set. These two images constitute genuine pair. The two presented derivatives $u_{x x}$ and $u_{y y}$ are calculated with averaging parameters defined by the 6 th $(s=5)$ and 8 th $(s=7)$ row of the table 11. White "+" and "-" mark local maximum and minimum points of the averaged Taylor expansion. The presented iris texture has no clearly expressed freckles, furrows, stripes or coronas in active sectors. However there are blurred dark and bright blobs where local maximum and minimum points indicate the blobs of the size that resonates with the averaging scale $\sigma$. 


\section{Similarity Metric}

Let us fix local features sets $A$ and $B$ formed from Taylor local extrema estimated at different scales. A particular feature $f_{i}=\left\{x_{i}, y_{i}, s_{i}, z_{i}\right\}$ has the following attributes:

- $\left(x_{i}, y_{i}\right)$ - local extremum position defined by two integer numbers $x_{i}$ and $y_{i}$.

- $s_{i} \in\{0,1, \cdots, 7\}$ - scale and filter type index (see table 1 for details).

- $z_{i} \in\{0,1\}$ - extremum type marker. 0 was used for local maximum points and 1 for local minimum points.

The similarity metric between two local features sets is defined by a normalized sum of similarity scores of feature pairs. A particular pair $\left(f_{i}^{A}, f_{j}^{B}\right)$ can gain non-zero similarity scores only if $f_{i}^{A}$ and $f_{j}^{B}$ correspond to the same filter and extremum type. Such features will be further called congeneric features. Two congeneric features can gain positive similarity only if their positions are sufficiently close. Distance between two affine $\left(x_{i}^{A}, y_{i}^{A}\right)$ and $\left(x_{j}^{B}, y_{j}^{B}\right)$ points is estimated considering possible alignment of two irises by means of an angular rotation and radial translation. Angular rotation and radial translation correspond to some shift along $x$ and $y$ directions respectively 1 . With a fixed $\alpha$ and $\beta$ shifts we use the following scores similarity expression:

$$
s\left(f_{i}^{A}, f_{j}^{B} ; \alpha, \beta\right)=\left\{\begin{array}{l}
0, \text { if } s_{i}^{A} \neq s_{j}^{B} \text { or } z_{i}^{A} \neq z_{j}^{B} \\
|D X-| x_{i}^{A}-x_{j}^{B}-\left.\alpha\right|_{+} \times \\
\times|D Y-| y_{i}^{A}-y_{j}^{B}-\beta||_{+}, \text {otherwise. }
\end{array}\right.
$$

Here $D X$ and $D Y$ are two positive integer parameters that define maximal acceptable distance along $x$ and $y$ directions and $|a|_{+}$operator assign 0 for negative $a$ values. The similarity scores between $A$ features set and $\alpha$ rotated and $\beta$ radially shifted $B$ set is defined as sum of the similarity scores of local features pairs, i. e.

$$
\operatorname{score}(A, B ; \alpha, \beta)=\sum_{i=1}^{N A} \sum_{j=1}^{N B} s\left(f_{i}^{A}, f_{j}^{B} ; \alpha, \beta\right) .
$$

Similarity scores between $A$ and $B$ features sets is defined by the following maximum:

$$
\operatorname{score}^{*}(A, B)=\max _{-K X \leq \alpha \leq K X,-K Y \leq \beta \leq K Y} \operatorname{score}(A, B ; \alpha, \beta) .
$$

Here $K X$ and $K Y$ are integer parameters defining $2 K X+1$ rotations and $2 K Y+1$ radial shifts under which similarity scores of shifted unwarped irises are optimized. They can be adjusted according to the database characteristics, for example with irises rotated in plane for up to $35^{\circ}, K X=25$ parameter value was used. Additionally, warped similarity technique from [6] was used. Finally, similarity score should be normalized according to the number of compared local features, for example divided by $N A+N B$.

\footnotetext{
${ }^{1}$ Shift along the $x$ direction inherits angular periodicity.
} 


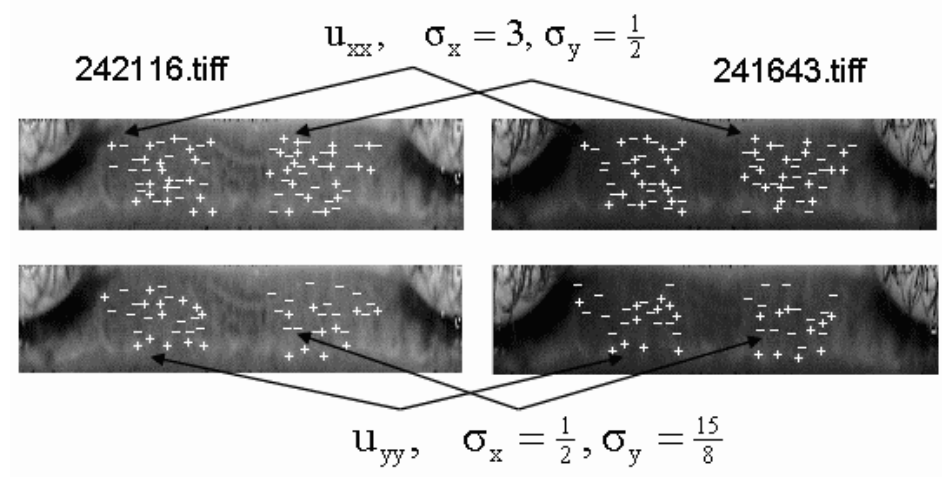

Fig. 2. Unwarped 242116 and 241643 iris images of the NIST ICE2005 data-set. "+" and "-" mark local maximums and minimums of averaged $u_{x x}$ and $u_{y y}$ derivatives ( $u_{x x}$ - above, $u_{y y}$ - below). Arrows points to the examples of extrema points that have correspondences in both images.

\section{Experimental Results}

We used four publicly available iris databases in all experiments: Chinese Academy of Sciences Casia 2.0 (device1) [8] (1200 images of 60 eyes), Casia 3.0 (interval) 8] (2655 images of 396 eyes), and US National Institute of Standards and Technology (NIST) "Iris Challenge Evaluation", experiment 1, (ICE1) 9] (1425 images of 128 eyes). Our implementation of fully automatic iris segmentation was applied to iris images in all databases. Experiments showed that verification quality increases as value of parameter $K$ (maximum number of congeneric features in one sector) increases. We used $K=7$ to present the results on all iris databases.

For Casia 2.0 (device 1) iris database ${ }_{20} C_{2} \times 60=11400$ genuine and ${ }_{60} C_{2} \times$ $20^{2}=708000$ impostor similarity scores were evaluated. It is recognized that Casia 2.0 iris database contains iris images of lower quality than Casia 3.0 [12]. Best result that can be found in the literature $E E R=0.58 \%$ is achieved by [12. We obtained $E E R=0.13 \%, Z \operatorname{eroF} A R=1.63 \%$, and ZeroFRR $=5.52 \%$ with proposed local extrema of averaged Taylor expansion coefficients as features (filled diamond marked curve in fig. 3).

For Casia 3.0 (interval) iris database 9023 genuine and 3514162 impostor similarity scores were evaluated. Impostor and genuine pairs are completely separable that's why direct estimation of Equal Error Rate (EER) is not possible. We approximated distributions of genuine and impostor empirical similarity scores by Gaussian distributions and concluded that proposed iris verification algorithm has $E E R=4.7 \times 10^{-2} \%$. Complete separation of impostor and genuine pairs was also received on Casia 1.0 7] database.

For the NIST "Iris Challenge Evaluation", experiment 1, (ICE-1) iris database local extrema based verification algorithm relatively produced the worst results. 
The image database contains very difficult and corrupted examples, sometimes with big eyelid occlusion, off-angle or with the iris partly outside the image frame. Poor focus of a part of the iris images especially degrade the quality of the verification that uses only information coming from local extrema. Out of focus iris images lost all subtle details of the iris texture that results in a significant decay of number of Taylor decomposition local extrema at tiny scales. For ICE-1 iris database 12214 genuine and 1002386 impostor similarity scores were evaluated. We obtained $E E R=0.25 \%$,frr@far $0.1=0.42 \%$,frr@far $0.01=0.83 \%$ (filled triangle marked curve in fig. 31).

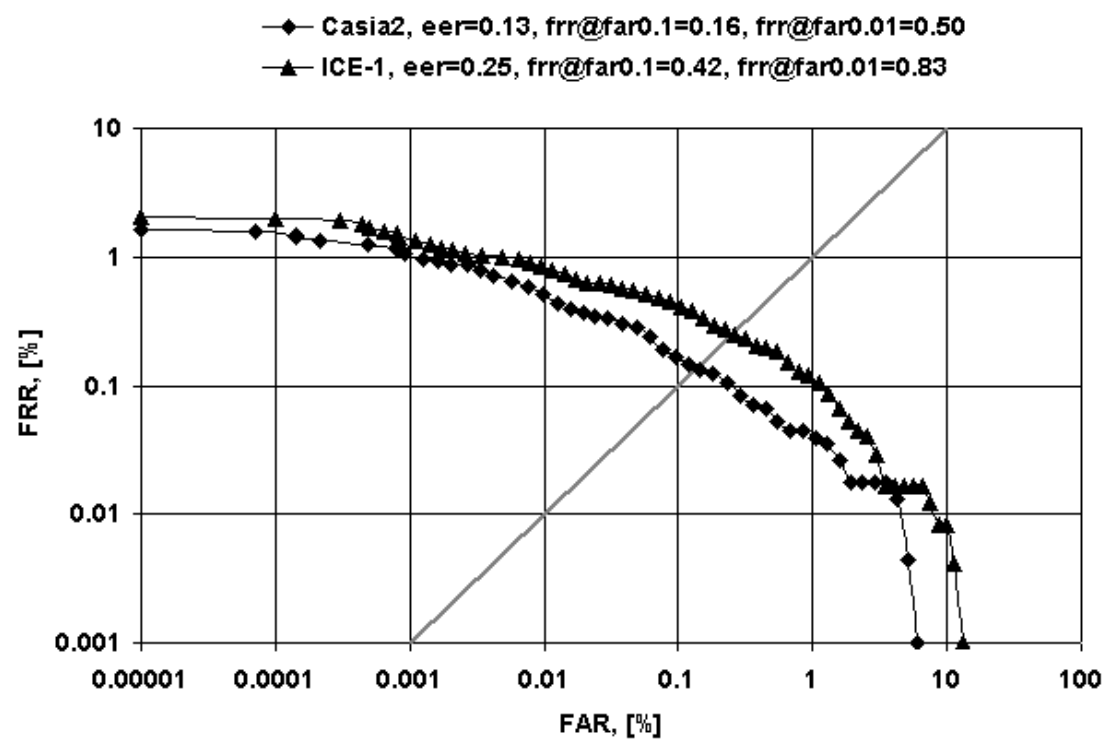

Fig. 3. DET curves of the proposed algorithm for Casia 2.0 (device 1) and ICE-1 iris databases

\section{Discussion}

The idea to use local extrema of wavelet, Gabor or other expansions for iris recognition is not new [13, 14, 15]. Unlike the 1D local extrema, which where used in [15], we use 2D local extremum points of the multiscale expansion. Such approach reduces the size or iris template and eliminates correlation of the local extremum points along radial direction. We chose the multiscale Taylor expansion since the first two coefficients of the expansion have a transparent interpretation and are associated with very computationally economic filters.

Although the authors of [16] recommends not to use Casia 1.0 data set for iris biometrics research because the images are edited "so that the pupil area is replaced by a circular region of uniform intensity", the images are still usable to 
test iris recognition performance (even if the segmentation of edited images is much easier). Our matching algorithm gives complete separation on the Casia 1.0 database. Additionally, we tested the proposed method on larger unedited Casia 3.0 (interval) database and received the same perfect separation of impostor and genuine pairs. About 10 state-of-the-art algorithms were tested on the NIST ICE1 database with the results available at [9]. Daugman's algorithm 2 [17] is one of the top performers of the ICE [9]. The proposed iris recognition algorithm is very close to Daugman's algorithm 1 [17] and is better than any other published algorithm that uses local extrema.

\section{Conclusion}

Iris features that can be extracted rapidly were selected on the base of Taylor multiscale expansion. A similarity measure that is robust to moderate iris segmentation inaccuracies for the chosen features was proposed. The proposed features demonstrate good verification performance on publicly available iris databases: Casia 2.0 (device 1), Casia 3.0 (interval) and NIST ICE-1.

Our iris template size is variable and in average contains $1.7 \mathrm{~Kb}$ (for $K=7$ ). Blurred, out of focus or simple texture containing iris images have reduced number of local extremum points. Therefore, DET curves can have increased ZeroFAR (i.e. FRR at the point where FAR equals to 0 ) that leads to increased slope near the ZeroFAR point, in the opposite to flat DET curves that are observed for phase based iris matching technique [18. One of the possible solutions could be additional attributes of the local extremum points that would decrease their matching probability.

Further research will be focused on fusing the phase based iris recognition algorithm (in our implementation) with the proposed method. The proposed method uses different features and different matching approach and is expected to be complimentary to phase based methods. Preliminary experiments showed extraordinary recognition quality results of the fused algorithm but other properties of the algorithm (speed, template size, fusing strategy etc.) must and will be addressed. Additionally, more publicly available databases will be used for testing iris recognition performance.

\section{References}

1. Daugman, J., Dowing, C.: Epigenetic randomness, complexity, and singularity of human iris patterns. In: Proceedings of the Royal Society, B, 268, Biological Sciences, pp. 1737-1740 (2001)

2. Daugman, J.: Statistical richness of visual phase information: update on recognizing persons by iris patterns. Int. J. Comput. Vis. 45(1), 25-38 (2001)

3. Daugman, J.: High confidence visual recognition of persons by a test of statistical independence. IEEE Transactions on Pattern Analysis and Machine Intelligence 15(11), 1148-1161 (1993) 
4. Daugman, J.: Results from 200 billion iris cross-comparisons. Technical Report UCAM-CL-TR-635 ISSN 1476-2986 (2005), http://www.cl.cam.ac.uk/techreports/UCAM-CL-TR-635.pdf

5. Aoptix: Breakthrough in iris recognition (2007), http://www. aoptix.com/pdf/BreakthroughBio.pdf

6. Bastys, A., Kranauskas, J., Masiulis, R.: Iris recognition by local extremum points of multiscale Taylor expansion (2008), http://dx.doi.org/10.1016/j.patcog.2008.12.004

7. Chinese Academy of Sciences - Institute of Automation Iris Database 1.0 (2003), http://www.sinobiometrics.com

8. Chinese Academy of Sciences - Institute of Automation Iris Database 3.0 (2005), http://www.cbsr.ia.ac.cn/IrisDatabase.htm

9. National Institute of Science and Technology (NIST): Iris Challenge Evaluation (2005), http://iris.nist.gov/ICE

10. Metz, C.E.: Basic principles of ROC analysis. Semin. Nucl. Med. 8, 283-298 (1978)

11. Monro, D.M., Rakshit, S., Zhang, D.: DCT-Based Iris Recognition. IEEE Transactions on Pattern Analysis and Machine Intelligence 29(4), 586-595 (2007)

12. Miyazawa, K., Ito, K., Aoki, T., Kobayashi, K., Nakajima, H.: A Phase-Based Iris Recognition Algorithm. In: Zhang, D., Jain, A.K. (eds.) ICB 2006. LNCS, vol. 3832, pp. 356-365. Springer, Heidelberg (2005)

13. Bae, K., Noh, S., Kim, J.: Iris feature extraction using independent component analysis. In: Proc. 4th Int. Conf. Audio- and Video-Based Biometric Person Authentication, pp. 838-844 (2003)

14. Ma, L.: Person identification based on iris recognition, Ph.D dissertation, Inst. Automation, Chinese Academy of Sciences, Beijing, China (2003)

15. Ma, L., Tan, T., Wang, Y., Zhang, D.: Efficient Iris Recognition by Characterizing Key Local Variations. IEEE Transactions on Image Processing 13(6) (2004)

16. Phillips, P.J., Bowyer, K.W., Flynn, P.J.: Comments on the CASIA version 1.0 Iris Data Set. IEEE Transactions on Pattern Analysis and Machine Intelligence 29(10), 1869-1870 (2007)

17. Daugman, J.: New Methods in Iris Recognition. IEEE Transactions on Systems, Man, and Cybernetics - Part B: Cybernetics 37(5), 1167-1175 (2007)

18. Daugman, J.: Flat ROC Curves, Steep Predictive Quality Metrics: Response to NISTIR-7440 and FRVT/ICE2006 Reports (2007), http://www.cl.cam.ac.uk/ jgd1000/Response_2_NIST_7440.pdf 